\section{Regards sur l'économie allemande}

Bulletin économique du CIRAC

75 | 2006

Varia

\title{
Budget européen : l'Allemagne se repositionne
}

\section{Markus Gabel}

\section{OpenEdition}

\section{Journals}

Édition électronique

URL : http://journals.openedition.org/rea/825

DOI : $10.4000 /$ rea. 825

ISBN : 978-2-8218-0846-1

ISSN : 1965-0787

Éditeur

CIRAC

Édition imprimée

Date de publication : 1 mars 2006

Pagination : 5-12

ISSN : 1156-8992

Référence électronique

Markus Gabel, « Budget européen : I'Allemagne se repositionne », Regards sur l'économie allemande [En ligne], 75 | mars 2006, mis en ligne le 24 juin 2008, consulté le 06 mai 2019. URL : http:// journals.openedition.org/rea/825; DOI : 10.4000/rea.825 


\title{
Budget européen : l'Allemagne se repositionne
}

\author{
Markus Gabel
}

Après avoir négocié pendant presque deux ans, les Etats membres sont parvenus mi-décembre 2005 à un accord sur le budget européen. Avec 862 milliards $€$, les dépenses prévues pour la période 2007-2013 correspondent à $1,045 \%$ du RNB de l'Union. Elles restent ainsi très au-dessous du 1,26 \% proposé initialement par la Commission. Sur le fond, les négociations ont abouti à des avancées peu perceptibles, mais pas moins fondamentales. II s'agit notamment de l'entrée dans un processus qui déplace successivement le curseur de la PAC vers l'objectif de compétitivité. L'Allemagne comme premier contributeur absolu pourra profiter de cette perspective. Or le cadre financier pour la période 2007-2013 marque également un certain retour à l'orthodoxie, celle de l'Allemagne comme premier contributeur net de l'UE. Ce retour à une charge plus élevée pour les finances publiques allemandes a été très habilement 'vendu' par le nouveau gouvernement outre-Rhin à une opinion publique qui commence juste à en comprendre l'ampleur. Le débat qui vient de débuter tourne autour de trois questions : d'abord celle des coûts et bénéfices des relations financières directes avec Bruxelles, ensuite celle de la structure des dépenses européennes et enfin celle de la juste place à donner au principe de subsidiarité en matière de politiques de subvention.

L'UE se finance essentiellement par des recettes versées par ses membres. Le Traité de la Communauté européenne dispose que « le budget est (...) intégralement financé par des ressources propres " (art. 269). Un financement par crédit est ainsi interdit. Au fil du temps, on constate une concentration des recettes - limitées à 1,24\% du RNB (Revenu National Brut) total de l'UE - sur deux principales sources. D'une part, la TVA qui provient de l'application d'un taux uniforme à l'assiette TVA de chaque Etat membre; pour 2004, ce taux était de $0,5 \%$, à appliquer à une assiette limitée à $50 \%$ du PNB du pays. D'autre part, la ressource PNB ; introduite en 1988, elle résulte de l'application d'un taux égal à la somme des PNB de tous les Etats membres, réparti au prorata de leur part dans le PNB de l'UE. Cette dernière est une ressource d'équilibre du budget : le taux est fixé de sorte à financer la différence entre le montant des dépenses et la somme des autres recettes. Pour l'année 2005, le poids des différentes ressources dans le total des recettes était de $74,5 \%$ pour la ressource PNB, de 14,1\% pour la TVA et de 11,4\% pour les ressources propres traditionnelles (prélèvements agricoles et droits de douanes).

\section{L’Allemagne, premier contributeur absolu}

La structure des recettes explique pourquoi, depuis dix ans, la part allemande dans le financement de l'UE a subi une longue glissade : elle est certes la plus élevée en valeur absolue (un peu plus de 22 milliards $€$ ), mais ce niveau n'a pas véritablement évolué depuis 1995 et a même nettement baissé en parts relatives, tombant de $29,2 \%$ à un peu plus de $21 \%$ en 2005 . Cette baisse s'explique par le double effet d'une consommation atone et d'une croissance inférieure à celle de la zone euro depuis plusieurs années. Ainsi, entre 1995 et 2004, la part allemande au PNB communautaire est passée d'un peu moins de

Contribution en baisse pour deux raisons :... 
$28,5 \%$ à un peu moins de $21,5 \%$-c'est presque à l'identique la part allemande dans le budget européen.

Evolution de la contribution allemande (en milliards $€$ )

\begin{tabular}{|c|c|c|c|c|c|c|c|c|c|c|}
\hline & 1996 & 1997 & 1998 & 1999 & 2000 & 2001 & 2002 & 2003 & 2004 & 2005 \\
\hline Budget européen (recettes) & 76,7 & 80,2 & 80,6 & 80,3 & 83,3 & 80,0 & 85,1 & 97,5 & 99,7 & 106,2 \\
\hline Financement allemand : & 224 & 226 & 202 & 205 & 207 & 19 & 19 & 224 & 201 & 2 5 \\
\hline - en \% & 29,2 & 28,2 & 25,1 & $\begin{array}{l}20,5 \\
25,5\end{array}$ & $\begin{array}{l}20,7 \\
24,8\end{array}$ & 24,4 & $\begin{array}{l}19,2 \\
22,6\end{array}$ & $\begin{array}{l}2<, 4 \\
23,0\end{array}$ & $\begin{array}{l}22,1 \\
22,1\end{array}$ & $\begin{array}{l}2<, 5 \\
21,2\end{array}$ \\
\hline
\end{tabular}

Source : Eurostat. Chiffres arrondis ; données provisoires pour 2005.

... baisse du La baisse relative de la contribution de l'Allemagne est d'abord la conséquence niveau de richesse... d'une baisse considérable du niveau de sa richesse par rapport à la moyenne européenne. Si on prend le groupe des principaux contributeurs au budget européen, l'Allemagne avait en 1984 un PNB par tête (en PPA, parité des pouvoirs d'achat) de près de $10 \%$ au-dessus de la moyenne européenne (France : 104 ; Pays-Bas : 95 ; Royaume Uni : 90,6). En 2003, juste avant l'élargissement, la situation s'est inversée : l'Allemagne n'atteint plus la moyenne européenne $(98,6)$ et se situe loin derrière le Royaume Uni $(111,2)$.

... et révision Ensuite, la situation de l'Allemagne s'est 'améliorée' notamment suite à la décide la contribution nette sion du Conseil européen de Berlin de 1999 visant à réduire à $25 \%$ du montant normal la participation de quatre contributeurs nets (Allemagne, Autriche, PaysBas et Suède) au financement du "chèque britannique ", une compensation accordée en 1984 au Royaume-Uni. Cette réduction est depuis financée par les autres Etats au prorata de leur part dans le PNB communautaire. Ainsi, la participation de la France au financement du rabais britannique est passée de $23 \%$ en 2001 à $30 \%$ en 2005.

La contribution absorbe $10 \%$ des recettes fiscales du Bund

Mais même si la contribution allemande a baissé relativement, elle reste une charge considérable, car elle est financée par le budget fédéral, plus exactement les recettes fiscales du Bund. En 2005, les 22 milliards € que l'Allemagne a versés à l'UE ont ainsi représenté près de $10 \%$ des recettes fiscales du Bund (les versements français représentent $7 \%$ des recettes fiscales de la France). II s'agit d'une contrainte importante pour le budget fédéral, compte tenu non seulement des règles du Pacte de stabilité et de croissance, mais également des futures charges budgétaires induites par le vieillissement de la population.

Le Bund abonde le budget de l'UE, les Länder bénéficient des aides

Contributeurs nets et bénéficiaires nets : des conflits programmés

Contribution nette de la RFA : $61,4 €$ par habitant
Cette contrainte est encore accentuée par le fait que contributeur et bénéficiaires se trouvent à des niveaux institutionnels différents : c'est le budget fédéral qui finance, mais ce sont essentiellement les Länder qui profitent des aides européennes. L'Allemagne se trouve ainsi régulièrement dans une position délicate lors des négociations budgétaires européennes : le gouvernement fédéral songe à contenir les dépenses (rappelons que 16 ans après la réunification, l'Allemagne verse toujours $4 \%$ de son PNB de l'ouest vers l'est), tandis qu'au niveau régional, les Länder, les riches comme les pauvres, ne cachent pas leur désir de recevoir le plus possible de fonds européens.

En plus des versements bruts, il est devenu habituel d'évaluer l'équité du budget européen en fonction de la contribution nette de chaque pays, c'est-à-dire du solde entre les versements à Bruxelles et les retours via les politiques communes, principalement la politique agricole et régionale. II est vrai que les écarts entre les pays en termes de contribution nette sont importants. Par ailleurs, le fameux " chèque britannique » a sérieusement biaisé la règle implicite du financement selon laquelle chaque Etat contribue selon sa richesse. Les clivages entre contributeurs nets et bénéficiaires nets expliquent la quasi-totalité des conflits entre Etats membres sur le budget depuis plus de vingt ans et ils ont largement pesé sur les négociations pour la période 2007-2013.

Depuis 2000, le nombre de contributeurs nets oscille entre huit et onze. Même si l'Allemagne supporte à nouveau le plus grand volume en termes absolus, elle 
ne prend pas la première place si ce solde est mis en relation avec le PNB (depuis 2002, les Pays-Bas supportent la contribution nette relative la plus importante). Et si on prend la contribution nette par tête, la position de l'Allemagne se relativise encore plus : ainsi, les Pays-Bas arrivent en première position avec $135,5 €$, devant le Luxembourg et la Suède avec respectivement $109,8 €$ et $83,4 €$; I'Allemagne n'est que cinquième avec 61,4€.

Evolution des contributions nettes *)

\begin{tabular}{|lcccccc|}
\hline & \multicolumn{2}{c}{2000} & \multicolumn{2}{c}{2002} & \multicolumn{2}{c}{$\mathbf{2 0 0 4}$} \\
Allemagne & en $\%$ & en $m r d . €$ & en $\%$ & en $m r d . €$ & en \% & en mrd. $€$ \\
Pays-Bas & $-0,42$ & $-8,28$ & $-0,24$ & $-5,07$ & $-0,36$ & $-7,65$ \\
Suède & $-0,39$ & $-1,54$ & $-0,51$ & $-2,19$ & $-0,43$ & $-1,96$ \\
Luxembourg & $-0,45$ & $-1,06$ & $-0,29$ & $-0,75$ & $-0,36$ & $-0,95$ \\
Belgique & $-0,28$ & $-0,06$ & $-0,25$ & $-0,05$ & $-0,28$ & $-0,06$ \\
Grande Bretagne & $-0,09$ & $-0,21$ & $-0,10$ & $-0,26$ & $-0,28$ & $-0,78$ \\
France & $-0,19$ & $-2,98$ & $-0,17$ & $-2,90$ & $-0,16$ & $-2,76$ \\
Autriche & $-0,05$ & $-0,74$ & $-0,14$ & $-2,18$ & $-0,12$ & $-1,91$ \\
Danemark & $-0,22$ & $-0,48$ & $-0,11$ & $-0,23$ & $-0,15$ & $-0,34$ \\
Italie & 0,15 & 0,24 & $-0,09$ & $-0,17$ & $-0,11$ & $-0,21$ \\
Finlande & 0,11 & 1,21 & $-0,23$ & $-0,88$ & $-0,06$ & $-0,79$ \\
Espagne & 0,22 & 0,27 & $-0,01$ & $-0,00$ & $-0,00$ & $-0,02$ \\
Irlande & 0,91 & 5,35 & 1,29 & 8,87 & 1,21 & 8,73 \\
Portugal & 1,77 & 1,72 & 1,50 & 1,58 & 1,40 & 1,56 \\
Grèce & 1,95 & 2,17 & 2,14 & 2,69 & 2,66 & 3,48 \\
Pologne & 3,66 & 4,43 & 2,39 & 3,39 & 2,22 & 3,37 \\
\hline
\end{tabular}

Source : Commission européenne, Rapport sur les dépenses de l'UE 2004 par Etat membre, sept. 2005. *) Soldes budgétaires entre les versements dans le budget européen et les retours vers les pays respectifs rapportés au PNB. Les chiffres incluent le rabais britannique et sont corrigés des dépenses d'administration.

Cette focalisation sur le concept des contributions nettes est cependant très discutable. II a une certaine légitimité - non seulement à cause du caractère toujours fortement redistributif du budget européen, mais aussi parce que l'indicateur s'est imposé de facto comme un des objectifs des politiques nationales. II ne faut cependant pas oublier qu'il s'agit d'une vision particulièrement simpliste qui souffre de problèmes méthodologiques (par exemple l'attribution par pays des dépenses administratives) ; il n'existe ainsi pas de définition claire de la notion de 'contribution nette'. La Commission utilise l'indicateur après correction du rabais britannique et des dépenses d'administration attribuables aux différents Etats. Par exemple pour l'année 2000, cette méthode place l'Allemagne $(-0,42 \%)$ en deuxième position derrière la Suède $(-0,45 \%$; voir le tableau cidessus). Or si on prend les contributions nettes sans les corrections utilisées par la Commission, l'Allemagne se trouve alors en troisième position $(-0,56 \%)$, loin derrière les Pays-Bas $(-0,80 \%)$ et même la Suède $(-0,57 \%)$.

Mais le principal problème des contributions nettes est qu'elles ne prennent pas assez en compte l'aspect de l'utilisation des dépenses européennes. Certaines politiques communes ont le caractère de biens collectifs. La valeur ajoutée créée par exemple par la politique extérieure commerciale, la politique commune de défense, ainsi que, sous certains aspects, la politique de cohésion, dépassent le volume des contributions nationales dans la mesure où il serait plus coûteux pour les Etats membres de réaliser l'effet recherché par leurs seules capacités nationales. C'est généralement le cas des petits pays, par exemple en ce qui concerne les politiques de cohésion ou de défense.

L'Allemagne, avec ses entreprises championnes à l'exportation, profite indéniablement beaucoup du marché commun. Mais elle perçoit également des sommes considérables du budget européen. En 2004, avec 11,7 milliards $€$, elle était le troisième bénéficiaire en termes absolus (ce qui correspond à $11,5 \%$ des dépenses européennes; presque autant en 2003). Seules l'Espagne $(16,4$ milliards $€)$ et la France $(12,9$ milliards $€)$ ont perçu davantage.
La contribution nette est une notion mal définie

Elle ne tient pas compte des effets de l'utilisation des dépenses

L'Allemagne perçoit plus au titre de la PAC que des aides régionales 
Dans ce contexte, il est peu connu que l'Allemagne reçoit plus au titre de la PAC qu'au titre des politiques régionales : environ 6 milliards $€$ sont allés dans le secteur agricole, la politique régionale ne bénéficiant que de 4,5 milliards $€$ (FEDER et FSE). La plupart des moyens pour la politique structurelle ont été alloués aux nouveaux Länder dans le cadre des mesures de l'Objectif-1 (régions avec un PIB par tête de moins de $75 \%$ de la moyenne européenne).

Qui bénéficie de combien sur le budget européen ? ${ }^{1)}$

\begin{tabular}{|c|c|c|c|c|c|c|c|c|c|c|c|}
\hline & en mrd. $€$ & en $\%^{2)}$ & $P N B^{3)}$ & & en mrd. $€$ & en $\%^{2)}$ & $P^{\prime} B^{3)}$ & & en mrd. $\epsilon$ & en $\%^{2)}$ & $P^{\prime} B^{3)}$ \\
\hline ES & 16,4 & 17,8 & 2,1 & EL & 5,9 & 6,3 & 3,5 & NL & 2,1 & 2,3 & 0,4 \\
\hline FR & 12,9 & 14,1 & 0,8 & BE & 4,7 & 5,4 & 1,7 & AT & 1,6 & 1,8 & 0,7 \\
\hline DE & 11,7 & 12,8 & 0,5 & PT & 4,5 & 4,8 & 3,3 & DK & 1,5 & 1,7 & 0,6 \\
\hline IT & 10,4 & 11,3 & 0,8 & IE & 2,9 & 3,1 & 2,3 & SE & 1,4 & 1,6 & 0,5 \\
\hline UK & 7,3 & 7,8 & 0,3 & PL & 2,8 & 3,0 & 1,4 & $\mathbf{F I}$ & 1,4 & 1,5 & 0,9 \\
\hline
\end{tabular}

Source: Eurostat; DG Budget (2005), Rapport financier 2004. (1) Volume global de dépenses par Etat membre. Données pour 2004 pour les 15 premiers bénéficiaires absolus. Chiffres arrondis. (2) Par rapport à l'ensemble des dépenses européennes. (3) Dépenses allouées par rapport au PNB.

L'Allemagne bénéficie peu du budget européen

Ces chiffres absolus révèlent que les dépenses du budget européen ne reviennent que de manière très sous-proportionnée vers l'Allemagne. Seuls le Royaume-Uni et les Pays-Bas reçoivent encore moins en relation de leur PNB respectif. Ceci a une conséquence simple : puisque les recettes sont largement proportionnelles à la richesse nationale des Etats membres, le fait d'être contributeur ou bénéficiaire net est essentiellement déterminé en fonction des dépenses du budget européen. C'est la traduction directe de l'esprit de solidarité qui inspire l'UE (Solidargemeinschaft).

\section{Perspective financière 2007-2013 : l'Allemagne paie plus}

Accroître la capacité du budget européen à générer une véritable valeur ajoutée est la principale ambition sous-jacente à l'élaboration de la nouvelle perspective financière 2007-2013. C'est du moins celle affichée par la Commission lors de sa proposition budgétaire de 2004, inspirée de la "Stratégie de Lisbonne ", et dans laquelle elle a redéfini les priorités du budget européen autour de l'objectif de la compétitivité. Cette proposition était très largement au-dessus de la position défendue par la "coalition d'austérité » (Allemagne, Autriche, France, Pays-Bas, Royaume Uni et Suède) lors de la lettre de décembre 2003 dans laquelle ces Etats demandaient à limiter le budget à $1 \%$ des RNB, ce qui correspond aux budgets des années 2005 et 2006.

Panorama des propositions de budget 2007-2013 (en milliards $€$ )

\begin{tabular}{|c|c|c|c|c|}
\hline & $\begin{array}{l}\text { Commission } \\
\text { européenne } \\
(10-02-2004)\end{array}$ & $\begin{array}{c}\text { Proposition } \\
\text { luxembourgeoise } \\
(17-06-2005)\end{array}$ & $\begin{array}{l}\text { Proposition } \\
\text { britannique } \\
(14-12-2005)\end{array}$ & $\begin{array}{c}\text { Compromis du } \\
17-12-2005\end{array}$ \\
\hline Compétitivité (Stratégie de Lisbonne) & 132,7 & 72,0 & 72,0 & 72,1 \\
\hline Cohésion (fonds structurels) & 338,7 & 308,6 & 298,9 & 307,6 \\
\hline PAC et développement durable & 404,6 & 377,8 & 367,3 & 371,2 \\
\hline Citoyenneté, liberté, sécurité, justice & 24,7 & 11,0 & 10,3 & 10,3 \\
\hline Politique extérieure & 95,3 & 50,0 & 50,0 & 50,0 \\
\hline Dépenses administratives & 28,62 & 50,3 & 49,3 & 50,3 \\
\hline Fonds pour la Roumanie et la Bulgarie & 0,2 & 0,8 & 0,8 & 0,8 \\
\hline En \% du RNB communautaires & 1,26 & 1,06 & 1,03 & 1,045 \\
\hline
\end{tabular}

Source : Becker (2006), Eurostat.

Un compromis conforme à la position des contributeurs nets
Avec un total qui représente $1,045 \%$ des recettes nationales brutes (RNB), le compromis trouvé le 17 décembre 2005 est finalement très proche de la position des contributeurs nets. Le Parlement européen a certes rejeté ce compromis, mais comme le montre l'expérience des dernières négociations budgétaires entre le Parlement et les Etats membres, les ajustements finaux ont de fortes chances d'être minimes. Deux Etats surtout, le Royaume Uni et l'Alle- 
magne, ont fait des concessions. Le premier a lâché un peu de lest sur son fameux « rabais "; le deuxième a accepté de creuser sa contribution nette et de redevenir le plus grand contributeur net rapporté au PNB. Cependant, en cédant sur son rabais, dont la défense s'avère aussi extravagante que celle de la politique agricole commune, le Royaume Uni a renoncé à seulement 10,5 milliards $€$ sur 7 ans. II ne sort donc pas vraiment perdant des négociations car il a obtenu que le rabais ne baisse pas ; il a seulement accepté que celui-ci ne s'accroisse pas dans les années à venir de 1,5 milliard € par an, comme prévu par la réglementation en place.

Or, ce rabais, dans sa totalité, n'a plus lieu d'être. Cette ristourne se justifiait il y a 20 ans par le peu de bénéfice que tirait le Royaume Uni de la PAC et par le marasme économique dans lequel était plongé le pays. Or, non seulement l'agriculture s'est un peu développée outre-Manche, grâce à la PAC d'ailleurs, mais surtout, l'économie britannique est florissante depuis une dizaine d'années. Le Royaume Uni ne finance ainsi que 13,8\% du budget européen alors que son PNB représente $19 \%$ de celui de l'Union.

La concession allemande est d'une autre ampleur. A partir de 2007, la contribution nette annuelle de l'Allemagne passera à 10,4 milliards $€$, soit environ 2 milliards de plus qu'aujourd'hui. Ce chiffre s'explique par le double effet de l'augmentation des sommes versées pour financer l'élargissement aux dix nouveaux Etats membres et de la baisse des aides structurelles que l'Allemagne reçoit de I'UE pour les nouveaux Länder. L'Allemagne devient ainsi clairement premier contributeur net, bien que du côté du seul financement, sa part dans l'ensemble du financement du budget continue toujours à baisser ; il devrait se situer autour de $20 \%$ (21\% en 2005).

Rapportée au PNB, la contribution nette allemande passe à 0,43\% (contre une moyenne de $0,33 \%$ pour la période 2000-2006). Elle dépasse ainsi celle des Pays-Bas $(0,33 \%)$. C'est maintenant I'Italie qui, avec $0,38 \%$, est deuxième contributeur net pendant la période 2007-2013, suivie par la France et la Suède avec chacune $0,37 \%$. Mais si l'Allemagne est en tête des contributeurs nets, elle retrouve avec les 0,43\% un niveau qu'elle avait déjà fin des années 1990 . Et si on compare à la proposition initiale de la Commission, elle est même gagnante : la Commission avait prévu une hausse à 0,49 \%, voire à 0,54 \% (sans la correction du rabais britannique). Enfin, rappelons-le, à cause de la dualité entre le niveau fédéral (Bund) et régional (Länder), la notion de contribution nette n'a aucune signification pour le budget fédéral. Les cinq Länder de l'Est en revanche sont financièrement perdants. Néanmoins, cette perte n'est guère importante : sur l'ensemble de la période 2007-2013, l'aide structurelle européenne ne s'abaissera que de 18 (période 2000-2006) à 13,5 milliards $€$.

En contrepartie, la Commission accorde des phasing out plus longs aux Länder est-allemands. Et plus important encore : elle a renoncé à son projet de limiter radicalement l'aide structurelle aux régions les plus pauvres. A partir de 2007, l'aide sera toujours concentrée sur les régions Objectif-1; mais toutes les régions ne seront plus aidées de la même manière. L'aide sera concentrée sur celles dont le revenu par tête est inférieur à $45 \%$ de la moyenne de l'UE. Les grandes entreprises y peuvent obtenir des aides allant jusqu'à $50 \%$ du coût de l'investissement. Le taux de la subvention tombe à $30 \%$ pour les régions dont le revenu par tête se situe entre 60 et $75 \%$ de la moyenne de l'UE.

Jusqu'en 2004, toutes les régions est-allemandes tombaient dans la catégorie de l'aide maximale. A l'avenir, les régions de Halle, Leipzig et le sud-ouest du Brandebourg ne satisfont plus au critère de $75 \%$. Elles sont victimes de l'effet statistique dû à l'élargissement (baisse du revenu moyen). Mais ces régions profitent d'un phasing out plus généreux et continueront à bénéficier du soutien de $30 \%$ jusqu'à 2010, au lieu de 2009 comme c'était initialement prévu par la Commission. Par ailleurs, dans toutes les régions qui satisfont aux conditions
Le rabais britannique n'était plus justifié en l'état

L'Allemagne à nouveau premier contributeur net...

... mais à un niveau plus faible

Les Länder est-allemands continueront d'être aidés,...

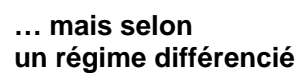


d'aide, le taux d'aide aux petites entreprises dépasse celui des grandes de 20 points de pourcentage (de 10 points pour les entreprises de taille moyenne).

\section{Repenser la structure du budget européen}

Réorientation du budget de l'UE vers la compétitivité...

Si on avait à définir aujourd'hui le budget de l'UE, personne ne dépenserait plus près de la moitié du budget pour l'agriculture (pour seulement $3 \%$ de la population) et plus d'un tiers pour la politique structurelle. Autant de ressources qu'on allouerait en priorité aux besoins de la recherche et de l'éducation. Les changements sur cette voie sont très lents ; mais ils sont perceptibles et plutôt robustes comme en témoigne l'évolution depuis 1988. Ainsi, le soutien au secteur agricole, même 'revalorisé' à travers le développement durable, est en perte de vitesse, et la ré-évaluation de la structure du budget comme elle vient d'être décidée pour 2008/09 ne changera certainement rien à cette tendance, au contraire. C'est ensuite l'ascension indéniable du soutien attribué aux politiques internes, et notamment de la compétitivité, qui constitue l'autre tendance forte depuis 1988, consolidée par le cadre financier pour 2007-2013.

Evolution de la structure budgétaire entre 1988 et $2013^{1)}$

\begin{tabular}{|c|c|c|c|c|}
\hline & $\begin{array}{c}\text { Delors I } \\
(1988-92)\end{array}$ & $\begin{array}{c}\text { Delors II } \\
(1993-99)\end{array}$ & $\begin{array}{c}\text { Agenda } 2000 \\
(2000-06)\end{array}$ & $\begin{array}{c}\text { Perspective } \\
2007-13^{2)}\end{array}$ \\
\hline Agriculture (PAC et développement durable) ${ }^{3)}$ & 58,1 & 48,2 & 46,1 & 43,1 \\
\hline Politique régionale (Cohésion) & 21,7 & 33,3 & 33,0 & 35,6 \\
\hline Politiques internes et compétitivité (recherche, justice etc.) & 3,8 & 6,0 & 6,8 & $9,6^{4)}$ \\
\hline Politique et actions extérieures & 5,1 & 6,9 & 8,8 & 5,8 \\
\hline Dépenses administratives & 9,3 & 4,8 & 5,2 & 5,8 \\
\hline Réserves (Fonds pour la Roumanie et la Bulgarie) & 2,0 & 0,8 & 0,2 & 0,0 \\
\hline
\end{tabular}

Source : Parlement européen. (1) en \% sur la base des moyennes annuelles de chaque période. (2) Entre parenthèses, les rubriques correspondantes du budget 2007/13. (3) Sur la base du compromis du 17 décembre 2005. (4) En valeur absolue, la rubrique compétitivité est dotée de 72 milliards $€$, dont 49,5 milliards $€$ pour la recherche et 8,4 milliards $€$ pour les réseaux transeuropéens. Ce sont des quantités toujours très faibles en comparaison des seules aides agricoles directes qui s'élèvent à 293,1 milliards $€$.

... et l'objectif de $3 \%$ de R\&D défini en 2000 à Lisbonne

Une politique de cohésion désormais controversée

En revenu par tête, l'Allemagne est onzième dans l'UE 25
Beaucoup d'efforts restent à faire pour que les objectifs de Lisbonne se concrétisent à l'horizon 2010 et que l'Europe voie sa croissance s'accélérer notablement, tirant l'emploi du fait de la recherche effectuée et des transferts technologiques opérés. L'objectif des $3 \%$ du PIB communautaire consacrés à la R\&D (Objectif de Lisbonne) apparaît encore lointain en Europe (1,99\% en 2002), alors qu'il est déjà réalisé au Japon (2,98\%) en 2000, et presque atteint aux Etats-Unis $(2,80 \%)$. Quelques exemples européens méritent cependant d'être cités : la Suède $(4,27 \%)$ et la Finlande $(3,49 \%)$. La France $(2,20 \%)$, quant à elle, se situe un peu au dessus de la moyenne européenne en 2002, derrière l'Allemagne (2,5\%), mais devant le Royaume Uni (1,84 \%).

Si un consensus est indéniablement en train d'émerger au sujet de la PAC et des politiques internes (pour une réduction de la première en faveur d'une extension de la seconde), c'est la politique de cohésion qui suscite le plus de controverses. L'élargissement de l'UE à 25 - conduisant à une amplification de sa part relative dans le budget 2007-2013 - n'a que retardé le débat. En Allemagne, il a déjà démarré, 'grâce' notamment à l'expérience de la reconstruction de l'ex-RDA, et l'argumentaire commence à se démarquer de celui de la Commission et de certains Etats membres.

L'UE ne justifie la politique régionale ni par des effets externes entre les régions (comme la pollution d'une zone agricole par la région industrielle voisine), ni par une assurance interrégionale contre des chocs asymétriques, mais uniquement par l'argument de la solidarité et de la compensation. L'objectif est de réduire les différences de bien-être entre les régions, mesurées en termes de revenu par tête. Ceux-ci divergent largement : un Luxembourgeois gagne plus de six fois le revenu d'un Letton, un Danois plus de quatre fois celui de son voisin letton. L'Allemagne, avec un revenu par tête de $110,2 \%$, se trouve en position 11 
dans l'UE-25, juste devant l'Italie, les pays de la cohésion (Espagne, Grèce et Portugal) et les nouveaux membres. Les régions allemandes les plus pauvres n'atteignent pas $75 \%$ de la moyenne européenne. Face à ces chiffres, et compte tenu de l'argumentaire de l'UE, il n'est guère compréhensible pourquoi l'Allemagne doit supporter la charge la plus forte au budget européen.

La Commission se base par ailleurs sur l'existence de la blue banana, c'est-àdire la région entre Londres et Milan qui abrite le centre de l'activité économique de l'UE, et ainsi les régions où les revenus par tête sont les plus élevés. Les régions plus pauvres se regroupent à la périphérie de cette banane. Dans son deuxième rapport sur la cohésion de 2001, la Commission souligne qu'elle considère que la plupart des régions allemandes se positionnent à l'intérieur de cette banane, justifiant ainsi la contribution élevée de l'Allemagne.

Reste l'épineuse question de l'efficacité des aides régionales. Dans un contexte où non seulement le nouveau gouvernement allemand s'est engagé à réduire fortement ses subventions, mais où au niveau européen, chaque subvention à une entreprise est vue d'un oeil très critique par la Commission, les subventions régionales ne doivent pas échapper à un test d'efficacité. Elles n'ont finalement une raison d'être que si la redistribution qu'elles entraînent est plus qu'un jeu à somme nulle, c'est-à-dire si les gains dans les régions aidées dépassent les pertes financières dans les régions riches. Jusqu'à ce jour, aucune étude globale n'a clairement pu démontrer une telle supériorité. Au plan de la croissance de l'UE comme une entité globale, un tel effet paraît même peu probable, car la majorité des régions aidées restent des zones à faible croissance.

II est indéniable que l'aide régionale a soutenu le rattrapage des pays de la cohésion. Et elle le fera également pour les nouveaux membres. La question est cependant de savoir si elle est indispensable au rattrapage. Ceci est également peu probable, compte tenu de l'importance à accorder à l'appartenance au marché commun, à une politique fiscale qui soutient la croissance ainsi qu'à la stabilité macroéconomique. Dans ce cas, les régions aux coûts de main d'œuvre moins élevés attireront des sites de production, délocalisés ou pas hors des agglomérations. La politique régionale soutient ce processus d'attraction par le développement des infrastructures et par des aides directes aux entreprises. Mais à nouveau, des doutes quant à son efficacité sont de mise.

Pendant la période 2000-2006, le soutien régional s'est surtout focalisé sur des programmes nationaux de développement d'infrastructures, essentiellement de transport, et du niveau de formation. Ce focus se prolonge à partir de 2007 par exemple à l'aide du soutien aux réseaux trans-européens. La région frontalière germano-polonaise en est un exemple. Cependant, un accès plus facile à des agglomérations dynamiques risque également de renforcer le schéma centre et périphérie tel qu'il existe: l'approvisionnement de la périphérie à partir du centre (dans le cas allemand Berlin, Leipzig et Dresde) sera facilité, entraînant ainsi l'installation d'autres sites d'activité au centre sans augmenter le niveau de vie à la périphérie. L'investissement dans le niveau de formation peut avoir le même effet, surtout s'il s'agit de personnes hautement qualifiées qui sont relativement plus mobiles (brain drain). L'effet positif des programmes de formation est plus probable chez les plus faiblement qualifiés qui, s'ils restent installés à la périphérie, peuvent contribuer à y augmenter le niveau de vie.

L'aide européenne aux régions d'Allemagne de l'Est et d'ailleurs a ainsi des effets ambivalents. Mais elle a également des effets clairement négatifs qui tiennent à la manière de fixer le niveau des salaires en Europe. Le système de négociation collective des salaires entraîne un faible niveau de différenciation de ces derniers, ce qui est une des raisons du niveau plus élevé du chômage dans les zones périphériques. La réduction de ce chômage est en principe l'affaire des Etats membres. Or les aides européennes contrecarrent leurs efforts car elles contribuent à maintenir des salariés dans la périphérie et à empêcher une plus large différenciation des salaires. A long terme, les aides régionales
Mais la plupart de ses régions sont localisées dans la blue banana

L'efficacité des aides régionales n'est pas prouvée

Elles favorisent les délocalisations interrégionales...

...accroissent les disparités entre centre et périphérie...

... et peuvent pérenniser les disparités régionales, comme dans les Länder de l'est 
contribuent ainsi - dans un contexte de négociations salariales collectives - à maintenir les disparités régionales. Bien qu'elles soient éligibles aux subventions européennes, le développement des régions d'Allemagne de l'Est reste ainsi sous-optimal.

L'exemple de l'Aufbau Ost depuis la réunification montre qu'une politique de soupoudrage est dans ce contexte une stratégie qui n'est bénéfique que si elle réussit à développer une dynamique autonome parvenant à freiner le déclin démographique et la migration des facteurs les plus mobiles vers les régions plus dynamiques. Mais même dans ce cas, l'aide régionale n'est pas forcément positive - si elle est accompagnée d'un rattrapage salarial trop rapide, ainsi que d'un schéma de négociation salariale collective. Pour l'Europe, l'enseignement de cette expérience est que la politique régionale ne peut fonctionner que si elle est conçue en concert avec les autres politiques et institutions économiques. Tant que ceci reste impossible à cause de l'étroitesse de la marge opérationnelle des politiques européennes, l'aide régionale devrait se concentrer sur des zones à fort potentiel de croissance et laisser une place la plus large possible au principe de subsidiarité. C'est ce constat qui fonde la nouvelle approche allemande de la politique de cohésion.

APRES UN NIVEAU Des PLUS haUtS AU MILIEU DES ANNEes 1990, la contribution relative de l'Allemagne au budget européen a nettement baissé, en termes absolus, mais également en terme de contributions nettes. Cette baisse s'est ralentie, voire arrêtée avec le nouveau cadre budgétaire 2007-2013. Elle a été le résultat d'un choix délibéré du gouvernement allemand, affichant ainsi une nette solidarité avec les nouveaux Etats membres. L'Allemagne sera premier contributeur net au nouveau budget. Cette place, mais également celle en termes absolus, devrait s'accentuer avec la confirmation de l'accélération de la croissance allemande et de l'amélioration de la confiance des ménages. La hausse prévue de la TVA (de 16 à $19 \%$ ) au $1^{\text {er }}$ janvier 2007 grossira encore un peu plus le versement allemand vers le budget européen.

Cette évolution est un message encourageant pour l'Europe, car l'Allemagne signale ainsi qu'elle a les moyens de jouer un rôle à nouveau plus actif. Le nouveau gouvernement a prononcé sa volonté de saisir cette opportunité, la présidence allemande de 2007 lui en donnera l'occasion. Mise à part la question du traité constitutionnel, c'est la réflexion concernant la structure et l'efficacité des aides européennes qui sera au centre de cette présidence. Le contrat de coalition de novembre 2005 en annonce les grandes lignes. II s'engage à réduire la concurrence fiscale injuste en Europe : celle résultant de la propension de certains Etats membres à demander d'un côté plus de subventions européennes et, de l'autre, à ne pas faire suffisamment d'efforts pour améliorer leur propre base fiscale. Ainsi, la nouvelle coalition à Berlin exprime son intention de réduire les aides régionales versées aux pays qui ne satisfont pas à un niveau minimum de recettes fiscales par rapport à leur niveau d'activité économique - un argument déjà évoqué pour des affaires intra-allemandes quant il s'est agi de réformer le fédéralisme financier.

\section{Indications bibliographiques :}

BECKER P., « Mehr Geld für Europa », Stiftung Wissenschaft und Politik, SWP-Aktuell, n4, janvier 2006

BeRTHOLd N., Neumann M., « Zahlt Deutschland drauf ? », ifo-Schnelldienst, n¹0, 2005

COMmission eUROPEenNe, Rapport sur les dépenses de l'UE 2004 par pays membre, sept. 2005, Troisième rapport sur la cohésion, 2004, et Im Dienst der Regionen, 2004 DG Budget (2005), Rapport financier 2004

GABEL M., "La réforme du fédéralisme financier : beaucoup de bruit pour rien », Regards sur l'économie allemande, $\mathrm{n}^{\circ} 52-53,2001$, et “ Aufbau Ost : entre désillusion, dilemme et renouveau, Regards sur l'économie allemande, $\mathrm{n}^{\circ} 71,2005$

Heinemann F., EU-Finanzplanung 2007-2013, Bertelsmann Stiftung, coll. Europa Vordenken , juin 2005. 